\title{
CO-C.E. SPHERES AND CELLS IN COMPUTABLE METRIC SPACES
}

\author{
ZVONKO ILJAZOVIĆ \\ Department of Mathematics, Faculty of Science, University of Zagreb, Bijenička 30, 10000 Zagreb, \\ Croatia \\ e-mail address: zilj@math.hr
}

\begin{abstract}
We investigate conditions under which a co-computably enumerable set in a computable metric space is computable. Using higher-dimensional chains and spherical chains we prove that in each computable metric space which is locally computable each co-computably enumerable sphere is computable and each co-c.e. cell with co-c.e. boundary sphere is computable.
\end{abstract}

\section{INTRODUCTION}

A closed subset of $\mathbb{R}^{m}$ is said to be computable if it can be effectively approximated by a finite set of points with rational coordinates with arbitrary given precision on arbitrary given bounded region of $\mathbb{R}^{m}$. A closed subset of $\mathbb{R}^{m}$ is said to be co-computable enumerable (co-c.e.) if its complement can be effectively covered by open balls. Each computable set is co-c.e. On the other hand, there exist co-c.e. sets which are not computable. In fact, while each nonempty computable set contains computable points, there exists a nonempty co-c.e. set which contains no computable points $([9])$. Although the implication

$$
S \text { co-computably enumerable } \Rightarrow S \text { computable }
$$

does not hold in general, there are certain conditions under which it does hold. The following result has been proved in [7]:

(i) if $S \subseteq \mathbb{R}^{m}$ is homeomorphic to $S^{n}$, where $S^{n} \subseteq \mathbb{R}^{n+1}$ is the unit sphere, then (1.1) holds;

(ii) if $S \subseteq \mathbb{R}^{m}$ is such that there exists a homeomorphism $f: B^{n} \rightarrow S$, where $B^{n} \subseteq \mathbb{R}^{n}$ is the unit ball, such that $f\left(S^{n-1}\right)$ is a co-c.e. set, then 1.1$)$ holds.

In the case $n=1$, i.e. in the case when $S$ is a topological circle or when $S$ is a co-c.e. arc with computable endpoints, the preceding result has been generalized in [5] to computable metric spaces with the effective covering property and compact closed balls. Furthermore, by [6], the assumption of the effective covering property and compact closed balls can be replaced here by the weaker assumption that a computable metric space is locally computable.

In this paper we prove that this result holds for every $m \geq 1$, i.e. we prove that if $(X, d, \alpha)$ is a computable metric space which is locally computable, then

1998 ACM Subject Classification: F.1.1, F.4.1, G.0.

Key words and phrases: computable metric space, computable set, co-c.e. set, $n$-chain, spherical $n$-chain.

DOI:10.2168/LMCS-7 (3:05) 2011

(C) Z. lljazović (c) Creative Commons 
(i) if $S$ is a co-c.e. set in $(X, d, \alpha)$ homeomorphic to $S^{n}$, then $S$ is computable in $(X, d, \alpha)$;

(ii) if $f: B^{n} \rightarrow S$ is a homeomorphism, where $S$ is co-c.e. in $(X, d, \alpha)$, such that $f\left(S^{n-1}\right)$ is also co-c.e., then $S$ is computable.

In order to prove this, we use techniques similar to those in [5]. In Section 3 we examine the topological side of the problem. We define the notions of $n$-chain and spherical $n$-chain in a metric space. These notions play the same role as the notions of a chain and a circular chain play in the proof of the main results of [5]. However, the higher-dimensional aspect of the problem will require some deeper topological facts and we will rely here on a result proved in [4]. In Section 4 we include computability into consideration and we prove the main results of the paper: Theorem 4.8 and Theorem 4.9 .

\section{Preliminaries}

If $X$ is a set, let $\mathcal{P}(X)$ denote the set of all subsets of $X$.

For $m \in \mathbb{N}$ let $\mathbb{N}_{m}=\{0, \ldots, m\}$. For $n \geq 1$ let

$$
\mathbb{N}_{m}^{n}=\left\{\left(x_{1}, \ldots, x_{n}\right) \mid x_{1}, \ldots, x_{n} \in \mathbb{N}_{m}\right\} .
$$

We say that a function $\Phi: \mathbb{N}^{k} \rightarrow \mathcal{P}\left(\mathbb{N}^{n}\right)$ is computable if the function $\bar{\Phi}: \mathbb{N}^{k+n} \rightarrow \mathbb{N}$ defined by

$$
\bar{\Phi}(x, y)=\chi_{\Phi(x)}(y)
$$

$x \in \mathbb{N}^{k}, y \in \mathbb{N}^{n}$ is computable (i.e. recursive). Here $\chi_{S}: \mathbb{N}^{n} \rightarrow\{0,1\}$ denotes the characteristic function of $S \subseteq \mathbb{N}^{n}$. A function $\Phi: \mathbb{N}^{k} \rightarrow \mathcal{P}\left(\mathbb{N}^{n}\right)$ is said to be computably bounded if there exists a computable function $\varphi: \mathbb{N}^{k} \rightarrow \mathbb{N}$ such that $\Phi(x) \subseteq \mathbb{N}_{\varphi(x)}^{n}$, for all $x \in \mathbb{N}^{k}$.

We say that a function $\Phi: \mathbb{N}^{k} \rightarrow \mathcal{P}\left(\mathbb{N}^{n}\right)$ is c.c.b. if $\Phi$ is computable and computably bounded.

\section{Proposition 2.1.}

(1) If $\Phi, \Psi: \mathbb{N}^{k} \rightarrow \mathcal{P}\left(\mathbb{N}^{n}\right)$ are c.c.b. functions, then the sets $\left\{x \in \mathbb{N}^{k} \mid \Phi(x)=\Psi(x)\right\}$ and $\left\{x \in \mathbb{N}^{k} \mid \Phi(x) \subseteq \Psi(x)\right\}$ are decidable.

(2) Let $\Phi: \mathbb{N}^{k} \rightarrow \mathcal{P}\left(\mathbb{N}^{n}\right)$ and $\Psi: \mathbb{N}^{n} \rightarrow \mathcal{P}\left(\mathbb{N}^{m}\right)$ be c.c.b. functions. Let $\Lambda: \mathbb{N}^{k} \rightarrow \mathcal{P}\left(\mathbb{N}^{m}\right)$ be defined by

$$
\Lambda(x)=\bigcup_{z \in \Phi(x)} \Psi(z)
$$

$x \in \mathbb{N}^{k}$. Then $\Lambda$ is a c.c.b. function.

(3) Let $\Phi: \mathbb{N}^{k} \rightarrow \mathcal{P}\left(\mathbb{N}^{n}\right)$ be c.c.b. and let $T \subseteq \mathbb{N}^{n}$ be c.e. Then the set $S=\left\{x \in \mathbb{N}^{k} \mid\right.$ $\Phi(x) \subseteq T\}$ is c.e.

A function $F: \mathbb{N}^{k+1} \rightarrow \mathbb{Q}$ is called computable if there exist computable functions $a, b, c:$ $\mathbb{N}^{k+1} \rightarrow \mathbb{N}$ such that

$$
F(x)=(-1)^{c(x)} \frac{a(x)}{b(x)+1}
$$

for each $x \in \mathbb{N}^{k+1}$. A number $x \in \mathbb{R}$ is said to be computable if there exists a computable function $g: \mathbb{N} \rightarrow \mathbb{Q}$ such that $|x-g(i)|<2^{-i}$ for each $i \in \mathbb{N}$.

By a computable function $\mathbb{N}^{k} \rightarrow \mathbb{R}$ we mean a function $f: \mathbb{N}^{k} \rightarrow \mathbb{R}$ for which there exists a computable function $F: \mathbb{N}^{k+1} \rightarrow \mathbb{Q}$ such that

$$
|f(x)-F(x, i)|<2^{-i}
$$


for all $x \in \mathbb{N}^{k}$ and $i \in \mathbb{N}$.

In the following proposition we state some elementary facts about computable functions $\mathbb{N}^{k} \rightarrow \mathbb{R}$.

\section{Proposition 2.2.}

(1) If $f, g: \mathbb{N}^{k} \rightarrow \mathbb{R}$ are computable, then $f+g, f-g: \mathbb{N}^{k} \rightarrow \mathbb{R}$ are computable.

(2) If $f: \mathbb{N}^{k} \rightarrow \mathbb{R}$ and $F: \mathbb{N}^{k+1} \rightarrow \mathbb{R}$ are functions such that $F$ is computable and $|f(x)-F(x, i)|<2^{-i}, \forall x \in \mathbb{N}^{k}, \forall i \in \mathbb{N}$, then $f$ is computable.

(3) If $f: \mathbb{N}^{n+1} \rightarrow \mathbb{R}$ and $\varphi: \mathbb{N} \rightarrow \mathbb{N}$ are computable functions, then the function $g: \mathbb{N} \rightarrow \mathbb{R}$ defined by

is computable.

$$
g(l)=\max _{0 \leq j_{1}, \ldots, j_{n} \leq \varphi(l)} f\left(l, j_{1}, \ldots, j_{n}\right)
$$

(4) If $f, g: \mathbb{N}^{k} \rightarrow \mathbb{R}$ are computable functions, then the set $\left\{x \in \mathbb{N}^{k} \mid f(x)>g(x)\right\}$ is c.e.

A tuple $(X, d, \alpha)$ is said to be a computable metric space if $(X, d)$ is a metric space and $\alpha: \mathbb{N} \rightarrow X$ is a sequence dense in $(X, d)$ (i.e. a sequence which range is dense in $(X, d)$ ) such that the function $\mathbb{N}^{2} \rightarrow \mathbb{R}$,

is computable (we use notation $\alpha=\left(\alpha_{i}\right)$ ).

$$
(i, j) \mapsto d\left(\alpha_{i}, \alpha_{j}\right)
$$

If $(X, d, \alpha)$ is a computable metric space, then a sequence $\left(x_{i}\right)$ in $X$ is said to be computable in $(X, d, \alpha)$ if there exists a computable function $F: \mathbb{N}^{2} \rightarrow \mathbb{N}$ such that

$$
d\left(x_{i}, \alpha_{F(i, k)}\right)<2^{-k}
$$

for all $i, k \in \mathbb{N}$. A point $a \in X$ is said to be computable in $(X, d, \alpha)$ if the constant sequence $a, a, \ldots$ is computable.

Let $(X, d, \alpha)$ be a computable metric space. Let $q: \mathbb{N} \rightarrow \mathbb{Q}$ be some fixed computable function whose image is $\mathbb{Q} \cap\langle 0, \infty\rangle$ and let $\tau, \tau^{\prime}: \mathbb{N} \rightarrow \mathbb{N}$ be some fixed computable functions such that $\left\{\left(\tau(i), \tau^{\prime}(i)\right) \mid i \in \mathbb{N}\right\}=\mathbb{N}^{2}$. For $i \in \mathbb{N}$ we define

$$
I_{i}=B\left(\alpha_{\tau(i)}, q_{\tau^{\prime}(i)}\right), \widehat{I_{i}}=\widehat{B}\left(\alpha_{\tau(i)}, q_{\tau^{\prime}(i)}\right) .
$$

Here, for $x \in X$ and $r>0$, we denote by $B(x, r)$ the open ball of radius $r$ centered at $x$ and by $\widehat{B}(x, r)$ the corresponding closed ball, i.e. $B(x, r)=\{y \in X \mid d(x, y)<r\}$, $\widehat{B}(x, r)=\{y \in X \mid d(x, y) \leq r\}$. For $A \subseteq X$ we will denote the closure of $A$ by $\bar{A}$.

As a consequence of Proposition 2.2 we get the following corollary.

Corollary 2.3. Let $(X, d, \alpha)$ be a computable metric space. The set $\left\{(k, i) \in \mathbb{N}^{2} \mid \alpha_{k} \in I_{i}\right\}$ is c.e.

A closed subset $S$ of $(X, d)$ is said to be computably enumerable in $(X, d, \alpha)$ if

$$
\left\{i \in \mathbb{N} \mid S \cap I_{i} \neq \emptyset\right\}
$$

is a c.e. subset of $\mathbb{N}$. A closed subset $S$ is said to be co-computably enumerable in $(X, d, \alpha)$ if there exists a computable function $f: \mathbb{N} \rightarrow \mathbb{N}$ such that

$$
X \backslash S=\bigcup_{i \in \mathbb{N}} I_{f(i)}
$$


It is easy to see that these definitions do not depend on functions $\tau, \tau^{\prime}$ and $q$. We say that $S$ is a computable set in $(X, d, \alpha)$ if $S$ is both computably enumerable and co-computably enumerable $([2,10])$.

Let $\sigma: \mathbb{N}^{2} \rightarrow \mathbb{N}$ and $\eta: \mathbb{N} \rightarrow \mathbb{N}$ be some fixed computable functions with the following property: $\{(\sigma(j, 0), \ldots, \sigma(j, \eta(j))) \mid j \in \mathbb{N}\}$ is the set of all finite sequences in $\mathbb{N}$ excluding the empty sequence, i.e. the set $\left\{\left(a_{0}, \ldots, a_{n}\right) \mid n \in \mathbb{N}, a_{0}, \ldots, a_{n} \in \mathbb{N}\right\}$. Such functions, for instance, can be defined using the Cantor pairing function. We use the following notation: $(j)_{i}$ instead of $\sigma(j, i)$ and $\bar{j}$ instead of $\eta(j)$. Hence

$$
\left\{\left((j)_{0}, \ldots,(j)_{\bar{j}}\right) \mid j \in \mathbb{N}\right\}
$$

is the set of all finite sequences in $\mathbb{N}$. For $j \in \mathbb{N}$ let $[j]$ be defined by

$$
[j]=\left\{(j)_{i} \mid 0 \leq i \leq \bar{j}\right\} .
$$

Note that the function $\mathbb{N} \rightarrow \mathcal{P}(\mathbb{N}), j \mapsto[j]$, is c.c.b.

Let $(X, d, \alpha)$ be a computable metric space. For $j \in \mathbb{N}$ we define

$$
J_{j}=\bigcup_{i \in[j]} I_{i}, \widehat{J}_{j}=\bigcup_{i \in[j]} \widehat{I}_{i} .
$$

The sets $J_{j}$ represent finite unions of rational balls and the sets $\widehat{J}_{j}$ finite unions of closed rational balls.

Corollary 2.4. Let $(X, d, \alpha)$ be a computable metric space. The set $\left\{(k, j) \in \mathbb{N}^{2} \mid \alpha_{k} \in J_{j}\right\}$ is c.e.

Proof. We have $\alpha_{k} \in J_{j}$ if and only if there exists $i \in \mathbb{N}$ such that $i \leq \bar{j}$ and $\alpha_{k} \in I_{(j)_{i}}$ and the claim follows from Corollary 2.3 .

A computable metric space $(X, d, \alpha)$ has the effective covering property if the set

$$
\left\{(w, j) \in \mathbb{N}^{2} \mid \widehat{I}_{w} \subseteq J_{j}\right\}
$$

is computably enumerable ([2]). It is not hard to see that this definition does not depend on the choice of the functions $q, \tau, \tau^{\prime}, \sigma, \eta$ which are necessary in the definitions of sets $I_{w}$ and $J_{j}$.

For example, if $\alpha: \mathbb{N} \rightarrow \mathbb{R}^{n}$ is a computable function (in the sense that the component functions of $\alpha$ are computable) whose image is dense in $\mathbb{R}^{n}$ and $d$ is the Euclidean metric on $\mathbb{R}$, then $\left(\mathbb{R}^{n}, d, \alpha\right)$ is a computable metric space. A sequence $\left(x_{i}\right)$ is computable in this computable metric space if and only if $\left(x_{i}\right)$ is a computable sequence in $\mathbb{R}^{n}$ and $\left(x_{1}, \ldots, x_{n}\right) \in$ $\mathbb{R}^{n}$ is a computable point in this space if and only if $x_{1}, \ldots, x_{n}$ are computable numbers. This computable metric space has the effective covering property (see e.g. [5]).

If $(X, d, \alpha)$ is a computable metric space, then a compact set $K$ in $(X, d)$ is said to be computably compact in $(X, d, \alpha)$ if $K$ is computably enumerable in $(X, d, \alpha)$ and if the set $\left\{j \in \mathbb{N} \mid K \subseteq J_{j}\right\}$ is c.e. ([1]). A computable metric space $(X, d, \alpha)$ is locally computable ([1]) if for each compact set $A$ in $(X, d)$ there exists a computably compact set $K$ in $(X, d, \alpha)$ such that $A \subseteq K$.

Let $(X, d, \alpha)$ be a computable metric space. A computable metric space $\left(Y, d^{\prime}, \beta\right)$ is said to be a subspace of $(X, d, \alpha)$ if $Y \subseteq X, d^{\prime}: Y \times Y \rightarrow \mathbb{R}$ is the restriction of $d: X \times X \rightarrow \mathbb{R}$ and $\beta$ is a computable sequence in $(X, d, \alpha)$.

The proofs of the following propositions can be found in [6]. 
Proposition 2.5. Let $\left(Y, d^{\prime}, \beta\right)$ be a subspace of a computable metric space $(X, d, \alpha)$ and let $S \subseteq Y$.

(i) If $S$ is co-c.e. in $(X, d, \alpha)$, then $S$ is co-c.e. in $\left(Y, d^{\prime}, \beta\right)$.

(ii) If $S$ is c.e. in $(X, d, \alpha)$, then $S$ is c.e. in $\left(Y, d^{\prime}, \beta\right)$. Conversely, if $S$ is closed in $(X, d)$ and c.e. in $\left(Y, d^{\prime}, \beta\right)$, then $S$ is c.e. in $(X, d, \alpha)$.

Proposition 2.6. Let $(X, d, \alpha)$ be a computable metric space and let $K$ be a nonempty compact set in $(X, d)$. Then $K$ is computably compact in $(X, d, \alpha)$ if and only if there exist a metric $d^{\prime}$ on $K$ and a sequence $\beta$ in $K$ such that $\left(K, d^{\prime}, \beta\right)$ is a subspace of $(X, d, \alpha)$ and $\left(K, d^{\prime}, \beta\right)$ has the effective covering property.

\section{3. $n$-CHAINS AND SPHERICAL $n$-CHAINS}

For $n \geq 1$ let

and

$$
B^{n}=\left\{x \in \mathbb{R}^{n} \mid\|x\| \leq 1\right\}
$$

$$
S^{n-1}=\left\{x \in \mathbb{R}^{n} \mid\|x\|=1\right\} .
$$

A topological space $X$ is called an $n$-cell if it is homeomorphic to $B^{n}$. We say that $X$ is an $n$-sphere if it is homeomorphic to $S^{n}$.

By the boundary sphere of an $n$-cell $E$ we mean the set $f\left(S^{n-1}\right)$, where $f: B^{n} \rightarrow E$ is a homeomorphism. (Note that the boundary sphere of $E$, when $E$ is a subspace of some topological space $X$, need not be equal to the topological boundary of $E$ in $X$.) The definition of the boundary sphere does not depend on a particular homeomorphism $f: B^{n} \rightarrow E$. Namely, this a consequence of the fact that each homeomorphism $B^{n} \rightarrow B^{n}$ maps $S^{n-1}$ onto $S^{n-1}$ (or equivalently $B^{n} \backslash S^{n-1}$ onto $B^{n} \backslash S^{n-1}$ ) which follows from the Invariance of domain theorem (see [8]): if $h: U \rightarrow \mathbb{R}^{n}$ is continuous and injective, where $U$ is an open subset of $\mathbb{R}^{n}$, then $h(U)$ is open.

The result that we want to prove can now be restated in this way: if $(X, d, \alpha)$ is a computable metric space which is locally computable, then

(1) each co-c.e. $n$-sphere is computable;

(2) each co-c.e. $n$-cell whose boundary sphere is co-c.e. is computable.

Let us first note that it is enough to prove this result in the case when $(X, d, \alpha)$ is a computable metric space which has the effective covering property and compact closed balls. Namely, suppose that the result holds for such computable metric spaces and let $(X, d, \alpha)$ be a computable metric space which is locally computable. Let $S \subseteq X$ be a co-c.e. $n$-sphere. Then $S \subseteq K$, where $K$ is computably compact in $(X, d, \alpha)$. By Proposition 2.6 there exist $d^{\prime}$ and $\beta$ such that $\left(K, d^{\prime}, \beta\right)$ is a subspace of $(X, d, \alpha)$ and such that $\left(K, d^{\prime}, \beta\right)$ has the effective covering property. By Proposition 2.5(i) $S$ is co-c.e. in $\left(K, d^{\prime}, \beta\right)$ and therefore $S$ is computable in $\left(K, d^{\prime}, \beta\right)$. Proposition 2.5(ii) implies now that $S$ is c.e. in $(X, d, \alpha)$, hence $S$ is computable in $(X, d, \alpha)$. In the same way we get that each co-c.e. $n$-cell in $(X, d, \alpha)$ whose boundary sphere is co-c.e. is computable.

Let us observe how the statement (2) was proved in [5] in the case $n=1$. Let $E$ be a co-c.e. arc with computable endpoints $a$ and $b$. For each $\varepsilon>0$ there exists a finite sequence of open sets $C_{0}, \ldots, C_{m}$ such that

(i) $E \subseteq C_{0} \cup \cdots \cup C_{m}$;

(ii) $a \in C_{0}, b \in C_{m}$; 
(iii) $C_{i} \cap C_{j}=\emptyset$ for all $i, j$ such that $|i-j|>1$;

(iv) each $C_{i}$ is the finite union of rational balls, i.e. it is equal to some $J_{j}$;

(v) $\operatorname{diam} C_{i}<\varepsilon$,

where $\operatorname{diam} C_{i}$ denotes the diameter of the set $C_{i}$ (see Figure 1). Since $S$ is co-c.e. and $(X, d, \alpha)$ has the effective covering property and compact closed balls, it is possible to find effectively for each $k \in \mathbb{N}$ sets $C_{0}, \ldots, C_{m}$ with properties (i)-(v), where $\varepsilon=2^{-k}$.

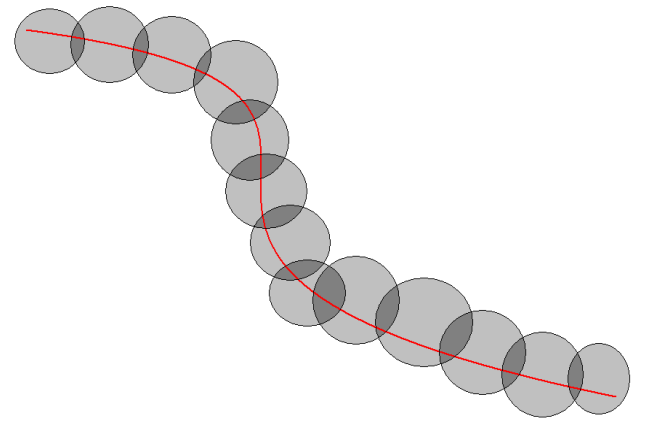

Figure 1.

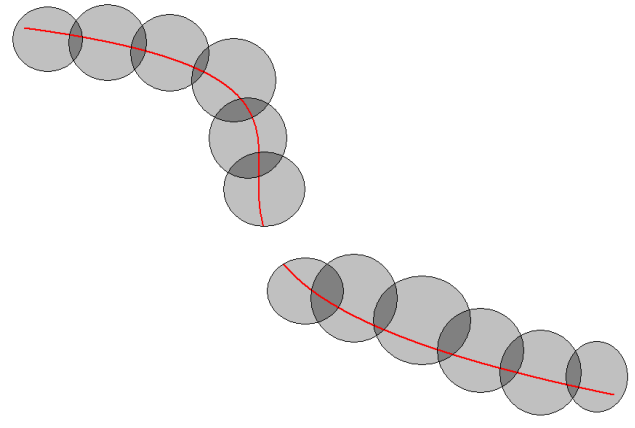

Figure 2.

However, this means that we can effectively approximate $S$, namely properties (i)-(v) imply that $C_{0} \cup \cdots \cup C_{m}$ is a $2^{-k}$-approximation of $S$ in the following sense: for each $x \in E$ there exists $y \in C_{0} \cup \cdots \cup C_{m}$ such that $d(x, y)<2^{-k}$ and for each $y \in C_{0} \cup \cdots \cup C_{m}$ there exists $x \in E$ such that $d(y, x)<2^{-k}$. Using this fact we can prove that $E$ is computable.

Why properties (i)-(v) imply that $C_{0} \cup \cdots \cup C_{m}$ is an $2^{-k}$-approximation of $S$ ? The fact that for each $x \in E$ there exists $y \in C_{0} \cup \cdots \cup C_{m}$ such that $d(x, y)<2^{-k}$ follows trivially from (i). On the other hand, the fact that for each $y \in C_{0} \cup \cdots \cup C_{m}$ there exists $x \in E$ such that $d(y, x)<2^{-k}$ can be easily deduced from (v) and the fact that

$$
C_{i} \cap S \neq \emptyset \text { for each } i \in\{0, \ldots, m\} \text {. }
$$

But why (3.1) holds? If we assume $C_{i} \cap E=\emptyset$ for some $i \in\{0, \ldots, m\}$, then $0<i<m$ and $C_{0} \cup \cdots \cup C_{i-1}$ and $C_{i+1} \cup \cdots \cup C_{m}$ are two disjoint open sets (Figure 2.) which cover $E$ and each of them intersects $E$ which contradicts the fact that $E$ is connected.

Suppose now that $E$ is a $2-$ cell which is co-c.e. and whose boundary sphere is co-c.e. In order to prove that $E$ is computable, we would like to proceed similarly as in the case of an arc. Naturally, in this case we are trying to find sets $C_{i, j}, 0 \leq i, j \leq m$, which satisfy properties similar to properties (i) $-(\mathrm{v})$ with basic difference that instead of (iii) we require

$$
C_{i, j} \cap C_{i^{\prime}, j^{\prime}}=\emptyset \text { if }\left|i-i^{\prime}\right|>1 \text { or }\left|j-j^{\prime}\right|>1 \text {. }
$$




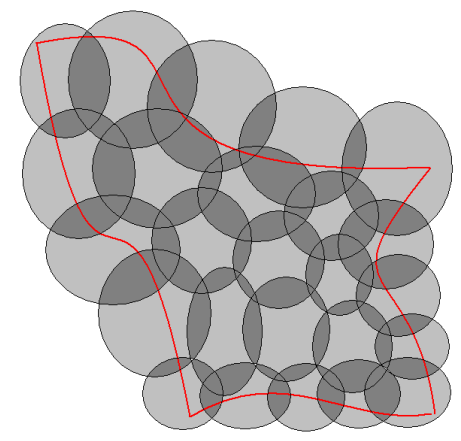

Figure 3. The sets $C_{i, j}$ cover the 2-cell whose boundary sphere is the red curve

The main question here is what other properties we should require so that those properties imply

$$
C_{i, j} \cap E \neq \emptyset \text { for all } i, j \in\{0, \ldots, m\} \text {; }
$$

the fact 3.3 is important since we want to conclude that $\bigcup_{i, j} C_{i, j}$ approximates $E$ in the same way as in the case of an arc.

If we suppose that $i_{0}$ and $j_{0}$ are such that $0<i_{0}<m, 0<j_{0}<m$ and such that $C_{i_{0}, j_{0}} \cap E=\emptyset$, then we cannot conclude in general that $E$ is covered by two disjoint open sets as in the case of an arc, but we can define the sets

$$
\begin{aligned}
& U=\bigcup_{i<i_{0}} \bigcup_{j} C_{i, j}, U^{\prime}=\bigcup_{i>i_{0}} \bigcup_{j} C_{i, j}, \\
& V=\bigcup_{j<j_{0}} \bigcup_{i} C_{i, j}, V^{\prime}=\bigcup_{j>j_{0}} \bigcup_{i} C_{i, j},
\end{aligned}
$$

and then these sets cover $E$ and we have $U \cap U^{\prime}=\emptyset, V \cap V^{\prime}=\emptyset$. (See Figure 4. The missing set is $C_{i_{0}, j_{0}}$. The vertical blue sets are $U$ and $U^{\prime}$, the horizontal blue sets are $V$ and $V^{\prime}$.)
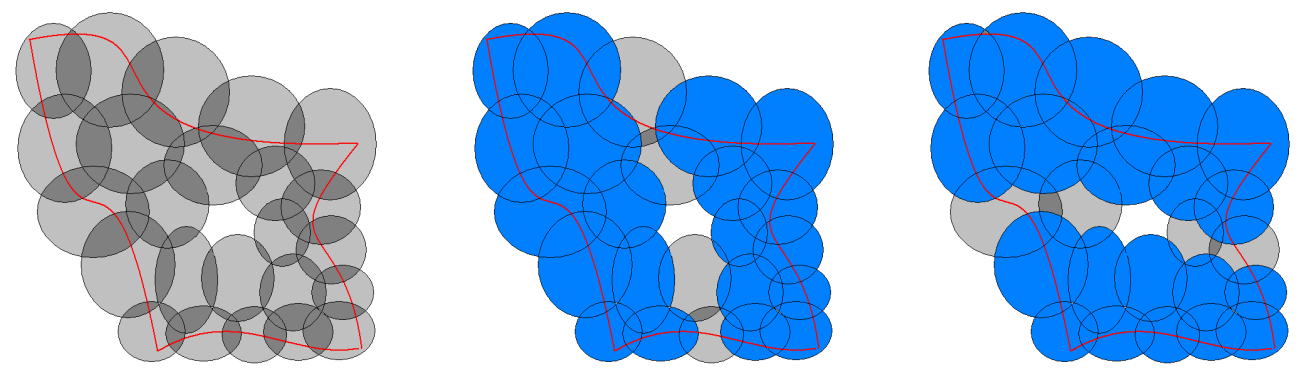

Figure 4.

Since $E$ is homeomorphic to $I^{2}=[0,1] \times[0,1]$, this raises the following question: is it possible to cover $I^{2}$ by open sets $U, U^{\prime}, V$ and $V^{\prime}$ so that $U \cap U^{\prime}=\emptyset, V \cap V^{\prime}=\emptyset$ and so that (see Figure 5.)

$$
\{0\} \times[0,1] \subseteq U,\{1\} \times[0,1] \subseteq U^{\prime},[0,1] \times\{0\} \subseteq V,[0,1] \times\{1\} \subseteq V^{\prime} ?
$$




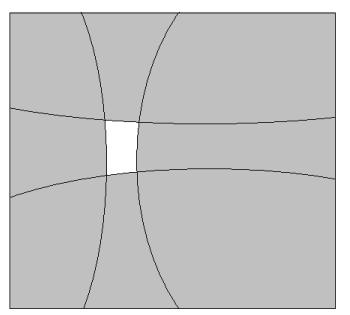

Figure 5.

Let $X$ be a topological space and let $A, B, L$ be subsets of $X$. We say that $L$ is a partition between $A$ and $B$ (see [4]) if there exist open sets $U$ and $W$ in $X$ such that

$$
A \subseteq U, B \subseteq W, U \cap W=\emptyset \text { and } X \backslash L=U \cup W .
$$

For $n \geq 1$ let

$$
I^{n}=\left\{\left(x_{1}, \ldots, x_{n}\right) \in \mathbb{R}^{n} \mid x_{1}, \ldots, x_{n} \in[0,1]\right\} .
$$

For $n \geq 1$ and $i \in\{1, \ldots, n\}$ let

$$
\begin{aligned}
& A_{i}^{n, 0}=\left\{\left(x_{1}, \ldots, x_{n}\right) \in I^{n} \mid x_{i}=0\right\}, \\
& A_{i}^{n, 1}=\left\{\left(x_{1}, \ldots, x_{n}\right) \in I^{n} \mid x_{i}=1\right\} .
\end{aligned}
$$

When the context is clear, we write $A_{i}^{0}$ and $A_{i}^{1}$ instead of $A_{i}^{n, 0}$ and $A_{i}^{n, 1}$. Let $\partial I^{n}$ denote the boundary of $I^{n}$ in $\mathbb{R}^{n}$, hence

$$
\partial I^{n}=A_{1}^{0} \cup \cdots \cup A_{n}^{0} \cup A_{1}^{1} \cup \cdots \cup A_{n}^{1} .
$$

It is a well known fact that there is a homeomorphism $h: B^{n} \rightarrow I^{n} \operatorname{such}$ that $h\left(S^{n-1}\right)=\partial I^{n}$. Hence if $E$ is an $n$-cell, then there is a homeomorphism $f: I^{n} \rightarrow E$. In this case $f\left(\partial I^{n}\right)$ is the boundary sphere of $E$.

The following theorem can be found in [4] (Theorem 1.8.1).

Theorem 3.1. Let $n \geq 1$. If $L_{i}$ is a partition between $A_{i}^{0}$ and $A_{i}^{1}$ in $I^{n}$ for $i \in\{1, \ldots, n\}$, then $\bigcap_{i=1}^{n} L_{i} \neq \emptyset$.

Corollary 3.2. Let $n \geq 1$. Suppose $U_{1}, \ldots, U_{n}$ and $V_{1}, \ldots, V_{n}$ are open subsets of $I^{n}$ such that

$$
U_{i} \cap A_{i}^{1}=\emptyset, V_{i} \cap A_{i}^{0}=\emptyset \text { and } U_{i} \cap V_{i}=\emptyset
$$

for all $i \in\{1, \ldots, n\}$. Then $I^{n} \neq U_{1} \cup \cdots \cup U_{n} \cup V_{1} \cup \cdots \cup V_{n}$.

Proof. Suppose the opposite. Then $\left\{U_{1}, \ldots, U_{n}, V_{1}, \ldots, V_{n}\right\}$ is an open cover of $I^{n}$ and let $\lambda$ be its Lebesgue number. We can certainly find finitely many closed subsets $B_{1}, \ldots, B_{l}$ of $I^{n}$ whose union is $I^{n}$ and each of which has the diameter less than $\lambda$. Then each of the sets $B_{1}, \ldots, B_{l}$ is contained in some of the sets $U_{1}, \ldots, U_{n}, V_{1}, \ldots, V_{n}$.

For $i \in\{1, \ldots, n\}$ we define $F_{i}^{0}$ to be the union of $A_{i}^{0}$ and all sets $B_{1}, \ldots, B_{l}$ which are subsets of $U_{i}$ and $F_{i}^{1}$ to be the union of $A_{i}^{1}$ all $B_{1}, \ldots, B_{l}$ which are subsets of $V_{i}$. Then $F_{1}^{0}, \ldots, F_{n}^{0}, F_{1}^{1}, \ldots, F_{n}^{1}$ are closed subsets of $I^{n}$, their union is $I^{n}$ and for each $i \in\{1, \ldots, n\}$ we have

$$
A_{i}^{0} \subseteq F_{i}^{0}, A_{i}^{1} \subseteq F_{i}^{1} \text { and } F_{i}^{0} \cap F_{i}^{1}=\emptyset
$$


Let $i \in\{1, \ldots, n\}$. Since $F_{i}^{0}$ and $F_{i}^{1}$ are closed and disjoint, there exist open sets $W_{i}^{0}$ and $W_{i}^{1}$ in $I^{n}$ which are disjoint and such that $F_{i}^{0} \subseteq W_{i}^{0}, F_{i}^{1} \subseteq W_{i}^{1}$. Let $L_{i}=I^{n} \backslash\left(W_{i}^{0} \cup W_{i}^{1}\right)$. Then $L_{i}$ is a partition between $A_{i}^{0}$ and $A_{i}^{1}$. We have

$$
\bigcap_{i=1}^{n} L_{i}=I^{n} \backslash \bigcup_{i=1}^{n}\left(W_{i}^{0} \cup W_{i}^{1}\right) \subseteq I^{n} \backslash \bigcup_{i=1}^{n}\left(F_{i}^{0} \cup F_{i}^{1}\right)=\emptyset,
$$

which is impossible by Theorem 3.1 .

Corollary 3.3. Let $n \geq 2$. Suppose $U_{1}, \ldots, U_{n-1}$ and $V_{1}, \ldots, V_{n-1}$ are open subsets of $\partial I^{n}$ such that

$$
U_{i} \cap\left(A_{i}^{n, 1} \cap A_{n}^{n, 0}\right)=\emptyset, V_{i} \cap\left(A_{i}^{n, 0} \cap A_{n}^{n, 0}\right)=\emptyset \text { and } U_{i} \cap V_{i}=\emptyset
$$

for all $i \in\{1, \ldots, n-1\}$. Let $E$ be the union of all $A_{i}^{\rho}$ such that $1 \leq i \leq n, \rho \in\{0,1\}$, $(i, \rho) \neq(n, 0)$, i.e.

$$
E=A_{1}^{n, 0} \cup \cdots \cup A_{n-1}^{n, 0} \cup A_{1}^{n, 1} \cup \cdots \cup A_{n}^{n, 1} .
$$

Then $E$ is not contained in the union $U_{1} \cup \cdots \cup U_{n-1} \cup V_{1} \cup \cdots \cup V_{n-1}$.
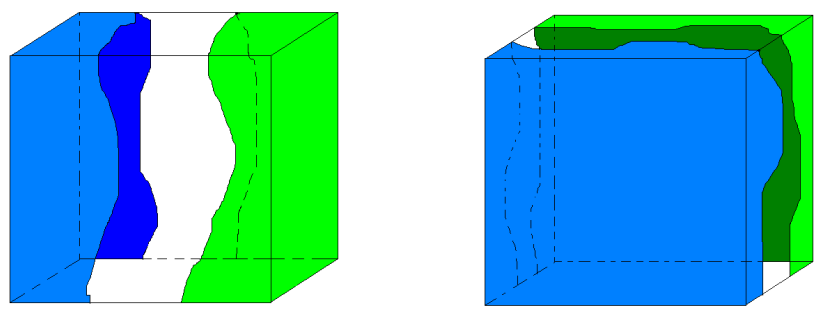

Figure 6 .

Proof. See Figure 6. (case $n=3$ ): the left and right blue sets are $U_{1}$ and $U_{2}$ respectively, and the left and right green sets are $V_{1}$ and $V_{2}$ respectively. In this figure $E$ equals the union of vertical faces of the cube and the upper face of the cube.

Let $f: E \rightarrow I^{n-1}$ be defined by

$$
f\left(x_{1}, \ldots, x_{n}\right)=\left(\frac{1}{2}+\frac{1}{2 x_{n}+1}\left(x_{1}-\frac{1}{2}\right), \ldots, \frac{1}{2}+\frac{1}{2 x_{n}+1}\left(x_{n-1}-\frac{1}{2}\right)\right) .
$$

It is straightforward to check that $f$ is bijective. Since $E$ is compact and $f$ clearly continuous, $f$ is a homeomorphism. For each $i \in\{1, . ., n-1\}$ we have

$$
f\left(A_{i}^{n, 0} \cap A_{n}^{n, 0}\right)=A_{i}^{n-1,0}, f\left(A_{i}^{n, 1} \cap A_{n}^{n, 0}\right)=A_{i}^{n-1,1} .
$$

Suppose that $E \subseteq U_{1} \cup \cdots \cup U_{n-1} \cup V_{1} \cup \cdots \cup V_{n-1}$. Then

$$
I^{n-1}=f\left(E \cap U_{1}\right) \cup \cdots \cup f\left(E \cap U_{n-1}\right) \cup f\left(E \cap V_{1}\right) \cup \cdots \cup f\left(E \cap V_{n}\right) .
$$

For each $i \in\{1, \ldots, n-1\}$ the sets $f\left(E \cap U_{i}\right)$ and $f\left(E \cap V_{i}\right)$ are open in $I^{n-1}$, disjoint and

$$
f\left(E \cap U_{i}\right) \cap A_{i}^{n-1,1}=\emptyset, f\left(E \cap V_{i}\right) \cap A_{i}^{n-1,0}=\emptyset .
$$

This is impossible by Corollary 3.2 . 
For $i \in\{1, \ldots, n\}$ let

$$
\partial_{i}^{0} \mathbb{N}_{m}^{n}=\left\{\left(x_{1}, \ldots, x_{n}\right) \in \mathbb{N}_{m}^{n} \mid x_{i}=0\right\}, \partial_{i}^{1} \mathbb{N}_{m}^{n}=\left\{\left(x_{1}, \ldots, x_{n}\right) \in \mathbb{N}_{m}^{n} \mid x_{i}=m\right\}
$$

and let

$$
\partial \mathbb{N}_{m}^{n}=\left(\bigcup_{1 \leq i \leq n} \partial_{i}^{0} \mathbb{N}_{m}^{n}\right) \cup\left(\bigcup_{1 \leq i \leq n} \partial_{i}^{1} \mathbb{N}_{m}^{n}\right)
$$

Let $X$ be a set, $n \geq 1$ and $m \in \mathbb{N}$. A function

$$
C: \mathbb{N}_{m}^{n} \rightarrow \mathcal{P}(X)
$$

is called an $n$-chain in $X$ (of length $m$ ) if

$$
C_{i_{1}, \ldots, i_{n}} \cap C_{j_{1}, \ldots, j_{n}}=\emptyset
$$

for all $\left(i_{1}, \ldots, i_{n}\right),\left(j_{1}, \ldots, j_{n}\right) \in \mathbb{N}_{m}^{n}$ such that $\left|i_{l}-j_{l}\right|>1$ for some $l \in\{1, \ldots, n\}$. Here we use $C_{i_{1}, \ldots, i_{n}}$ to denote $C\left(i_{1}, \ldots, i_{n}\right)$.

A spherical $(n-1)$-chain in $X$ (of length $m$ ) is a function

$$
C: \partial \mathbb{N}_{m}^{n} \rightarrow \mathcal{P}(X)
$$

such that (3.4) holds for all $\left(i_{1}, \ldots, i_{n}\right),\left(j_{1}, \ldots, j_{n}\right) \in \partial \mathbb{N}_{m}^{n}$ such that $\left|i_{l}-j_{l}\right|>1$ for some $l \in\{1, \ldots, n\}$.
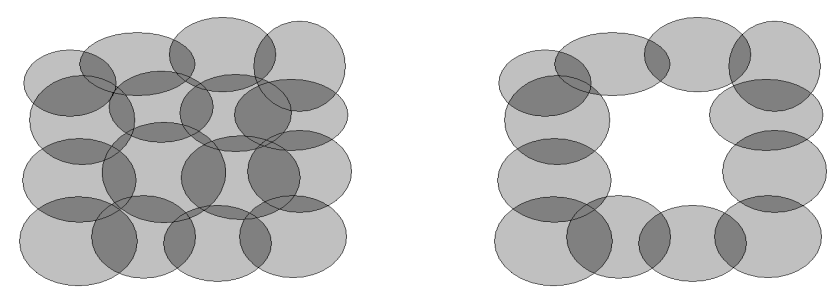

Figure 7. A 2-chain and a spherical 1-chain

If $C: \mathbb{N}_{m}^{n} \rightarrow \mathcal{P}(X)$ is a function, we define its boundary $\partial C$ as the restriction of $C$ to $\partial \mathbb{N}_{m}^{n}$. For $i \in\{1, \ldots, n\}$ and $\rho \in\{0,1\}$ we define $\partial_{i}^{\rho} C$ as the restriction of $C$ to $\partial_{i}^{\rho} \mathbb{N}_{m}^{n}$. Note: if $C$ is an $n$-chain, then $\partial C$ is a spherical $(n-1)$-chain.

If $C: \partial \mathbb{N}_{m}^{n} \rightarrow \mathcal{P}(X)$ is a function and $i \in\{1, \ldots, n\}, \rho \in\{0,1\}$, we also use $\partial_{i}^{\rho} C$ to denote the restriction of the function $C$ to $\partial_{i}^{\rho} \mathbb{N}_{m}^{n}$.

If $(X, d)$ is a metric space, then we say that an $n$-chain $C=\left(C_{i_{1}, \ldots, i_{n}}\right)_{0 \leq i_{1}, \ldots, i_{n} \leq m}$ in $X$ is open if $C_{i_{1}, \ldots, i_{n}}$ is an open set in $(X, d)$ for all $i_{1}, \ldots, i_{n} \in \mathbb{N}_{m}$. We similarly define the notion of a compact $n$-chain in $(X, d)$ and the notions of open spherical $n$-chain and compact spherical $n$-chain.

In general, if $A$ is a set and $f: A \rightarrow \mathcal{P}(X)$ a function, we will denote by $\bigcup f$ the union $\bigcup_{a \in A} f(a)$ and we will say that $f$ covers $S$, where $S \subseteq X$, if $S \subseteq \bigcup f$. If $(X, d)$ is a metric space and $f(a)$ a nonempty bounded set for each $a \in A$, then we define $\operatorname{mesh}(f)$ as the number

$$
\operatorname{mesh}(f)=\max _{a \in A}(\operatorname{diam} f(a)) .
$$

Let $\varepsilon>0$. A (spherical) $n$-chain $C$ in a metric space $(X, d)$ is said to be a (spherical) $\varepsilon-n$-chain if $\operatorname{mesh}(C)<\varepsilon$. 
A function $C: A \rightarrow \mathcal{P}(X)$, where $A \subseteq \mathbb{N}_{m}^{n}$, is said to be $\varepsilon$-proper if for all $\left(i_{1}, \ldots, i_{n}\right)$, $\left(j_{1}, \ldots, j_{n}\right) \in A$ such that $\left|i_{1}-j_{1}\right| \leq 1, \ldots,\left|i_{n}-j_{n}\right| \leq 1$ there exist $x \in C_{i_{1}, \ldots, i_{n}}$ and $y \in C_{j_{1}, \ldots, j_{n}}$ such that $d(x, y)<\varepsilon$.

The proof of the following lemma is straightforward.

Lemma 3.4. Let $(X, d)$ be a metric space, $\varepsilon>0$ and $A \subseteq \mathbb{N}_{m}^{n}$. Let $C, D: A \rightarrow \mathcal{P}(X)$ be such that $D(a) \neq \emptyset$ and $D(a) \subseteq C(a)$ for each $a \in A$. Suppose $C$ is $\varepsilon$-proper and $\operatorname{mesh}(C)<\varepsilon$. Then $D$ is $3 \varepsilon-$ proper.

Lemma 3.5. Let $(X, d)$ be a metric space and let $K$ be a compact (spherical) $n$-chain of length $m$ in $(X, d)$. Suppose $U_{1}, \ldots, U_{k}$ are open sets. Then there exists an open (spherical) $n$-chain $C$ of length $m$ in $(X, d)$ such that $K_{a} \subseteq C_{a}$ for all $a \in \mathbb{N}_{m}^{n}$ and such that $C_{a} \subseteq U_{i}$ whenever $i \in\{1, \ldots, k\}$ is such that $K_{a} \subseteq U_{i}$. Moreover, if $\operatorname{mesh}(K)<r$, we can choose $C$ so that $\operatorname{mesh}(C)<2 r$.

Proof. If $S \subseteq X$ and $\varepsilon>0$ let

$$
S_{\varepsilon}=\bigcup_{s \in S} B(s, \varepsilon) .
$$

This is clearly an open set. If $S$ is a compact set contained is some open set $V$, then there exists $\varepsilon>0$ such that $S_{\varepsilon} \subseteq V$. Furthermore, if $S$ and $T$ are disjoint compact sets, then there exists $\varepsilon>0$ such that $S_{\varepsilon} \cap T_{\varepsilon}=\emptyset$. It follows readily from this that there exists $\varepsilon>0$ such that $C: \mathbb{N}_{m}^{n} \rightarrow \mathcal{P}(X)$ (or $C: \partial \mathbb{N}_{m}^{n} \rightarrow \mathcal{P}(X)$ ) defined by $C_{a}=\left(K_{a}\right)_{\varepsilon}$ is a desired $n$-chain (spherical $n$-chain).

Proposition 3.6. Let $n \geq 2$. Suppose $f: \partial I^{n} \rightarrow S$ is a homeomorphism, where $S$ is a subspace of a metric space $(X, d)$. Let $U_{i}^{\rho}, 1 \leq i \leq n, \rho \in\{0,1\}$, be open sets in $(X, d)$ such that

$$
f\left(A_{i}^{\rho}\right) \subseteq U_{i}^{\rho}
$$

for all $i \in\{1, \ldots, n\}$ and $\rho \in\{0,1\}$. Then for each $\varepsilon>0$ there exists an open spherical $\varepsilon-(n-1)$-chain $C$ in $(X, d)$ which is $\varepsilon$-proper, which covers $S$ and such that

$$
f\left(A_{i}^{\rho}\right) \subseteq \bigcup\left(\partial_{i}^{\rho} C\right) \subseteq U_{i}^{\rho}
$$

for all $i \in\{1, \ldots, n\}$ and $\rho \in\{0,1\}$.

Proof. For $m \in \mathbb{N}$ let $D^{m}: \mathbb{N}_{m}^{n} \rightarrow \mathcal{P}\left(I^{n}\right)$ be defined by

$$
D_{i_{1}, \ldots, i_{n}}^{m}=\left[\frac{i_{1}}{m+1}, \frac{i_{1}+1}{m+1}\right] \times \cdots \times\left[\frac{i_{n}}{m+1}, \frac{i_{n}+1}{m+1}\right] .
$$

Then $D^{m}$ is a compact $n$-chain in $I^{n}$ which covers $I^{n}$. Clearly for each $\varepsilon>0$ there exists $m \in \mathbb{N}$ such that $\operatorname{mesh}\left(D^{m}\right)<\varepsilon$. Note that for all $\left(i_{1}, \ldots, i_{n}\right),\left(j_{1}, \ldots, j_{n}\right) \in \mathbb{N}_{m}^{n}$ such that $\left|i_{1}-j_{1}\right| \leq 1, \ldots,\left|i_{n}-j_{n}\right| \leq 1$ we have

$$
D_{i_{1}, \ldots, i_{n}}^{m} \cap D_{j_{1}, \ldots, j_{n}}^{m} \neq \emptyset .
$$

We easily conclude from this that for each $\varepsilon>0$ there exists $m \in \mathbb{N}$ such that $\operatorname{mesh}\left(D^{m}\right)<\varepsilon$ and such that $D^{m}$ is $\varepsilon$-proper.

The boundary $\partial D^{m}$ is a spherical $(n-1)$-chain in $I^{n}$ which covers $\partial I^{n}$.

For $m \in \mathbb{N}$ let $G^{m}: \partial \mathbb{N}_{m}^{n} \rightarrow \mathcal{P}\left(\partial I^{n}\right)$ be defined by

$$
G^{m}(a)=\left(\partial D^{m}\right)(a) \cap \partial I^{n} .
$$


Then $G^{m}$ is a compact spherical $(n-1)$-chain in $\partial I^{n}$ which covers $\partial I^{n}$, moreover

$$
A_{i}^{\rho} \subseteq \bigcup\left(\partial_{i}^{\rho} G^{m}\right)
$$

for all $i \in\{1, \ldots, n\}$ and $\rho \in\{0,1\}$. Note that these sets need not be equal, however:

$$
\text { for each } x \in \bigcup\left(\partial_{i}^{\rho} G^{m}\right) \text { there exists } y \in A_{i}^{\rho} \text { such that } d^{\prime}(x, y) \leq \frac{1}{m+1},
$$

where $d^{\prime}$ is the Euclidean metric on $\mathbb{R}^{n}$. We also have that for each $\varepsilon>0$ there exists $m \in \mathbb{N}$ such that $\operatorname{mesh}\left(G^{m}\right)<\varepsilon$ and such that $G^{m}$ is $\varepsilon$-proper.

For $m \in \mathbb{N}$ let $F^{m}: \partial \mathbb{N}_{m}^{n} \rightarrow \mathcal{P}(S)$ be defined by

$$
F^{m}(a)=f\left(G^{m}(a)\right) .
$$

Then $F^{m}$ is a compact spherical $(n-1)$-chain in $S$ which covers $S$ and such that

$$
f\left(A_{i}^{\rho}\right) \subseteq \bigcup\left(\partial_{i}^{\rho} F^{m}\right)
$$

for all $i \in\{1, \ldots, n\}$ and $\rho \in\{0,1\}$.
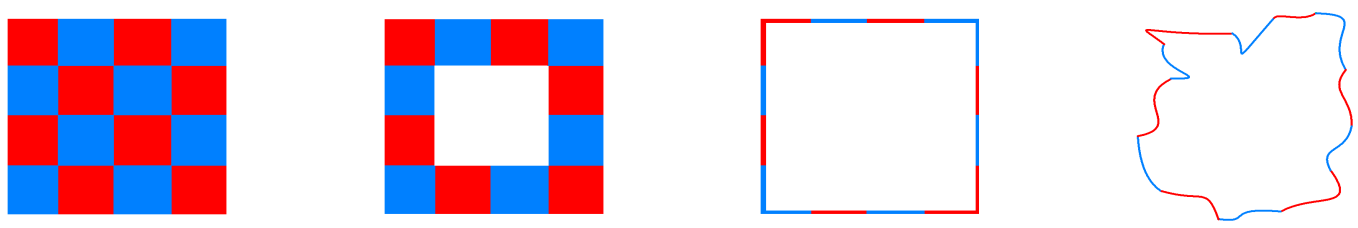

Figure 8. $D^{3}, \partial D^{3}, G^{3}$ and $F^{3}$ (in the case $n=2$ )

The fact that $f$ is uniformly continuous implies, together with $(3.5)$, that for each $\varepsilon>0$ there exists $m \in \mathbb{N}$ with the property that $F^{m}$ is $\varepsilon$-proper, $\operatorname{mesh}\left(F^{m}\right)<\varepsilon$ and with the property that for all $i \in\{1, \ldots, n\}, \rho \in\{0,1\}$ and $x \in \bigcup\left(\partial_{i}^{\rho} F^{m}\right)$ there exists $y \in f\left(A_{i}^{\rho}\right)$ such that $d(x, y)<\varepsilon$.

Let $\varepsilon>0$. Using the fact that the sets $A_{i}^{\rho}$ are compact and $U_{i}^{\rho}$ are open, it is not hard to conclude now that there exists $m \in \mathbb{N}$ such that $F^{m}$ is $\varepsilon$-proper, $\operatorname{mesh}\left(F^{m}\right)<\frac{\varepsilon}{2}$ and

$$
f\left(A_{i}^{\rho}\right) \subseteq \bigcup\left(\partial_{i}^{\rho} F^{m}\right) \subseteq U_{i}^{\rho}
$$

for all $i \in\{1, \ldots, n\}$ and $\rho \in\{0,1\}$. Now we apply Lemma 3.5 to $F$ and the sets $U_{i}^{\rho}$ and we get an open spherical $\varepsilon-(n-1)$-chain $C$ in $(X, d)$ which is $\varepsilon$-proper such that

$$
f\left(A_{i}^{\rho}\right) \subseteq \bigcup\left(\partial_{i}^{\rho} C\right) \subseteq U_{i}^{\rho}
$$

for all $i \in\{1, \ldots, n\}$ and $\rho \in\{0,1\}$.

In the same way we prove the following proposition.

Proposition 3.7. Let $n \geq 1$. Suppose $f: I^{n} \rightarrow E$ is a homeomorphism, where $E$ is a subspace of a metric space $(X, d)$. Let $U_{i}^{\rho}, 1 \leq i \leq n, \rho \in\{0,1\}$, be open sets in $(X, d)$ such that

$$
f\left(A_{i}^{\rho}\right) \subseteq U_{i}^{\rho}
$$

for all $i \in\{1, \ldots, n\}$ and $\rho \in\{0,1\}$. Then for each $\varepsilon>0$ there exists an open $\varepsilon-n$-chain $C$ in $(X, d)$ which is $\varepsilon$-proper, which covers $E$ and such that

$$
f\left(A_{i}^{\rho}\right) \subseteq \bigcup\left(\partial_{i}^{\rho} C\right) \subseteq U_{i}^{\rho}
$$

for all $i \in\{1, \ldots, n\}$ and $\rho \in\{0,1\}$. 
If $(X, d)$ is a metric space, then for nonempty subsets $S$ and $T$ of $X$ we denote the number $\inf \{d(x, y) \mid x \in S, y \in T\}$ by $d(S, T)$.

The next proposition provides conditions under which a spherical $(n-1)$-chain approximates an $(n-1)$-sphere.

Proposition 3.8. Let $f: \partial I^{n} \rightarrow S$ be a homeomorphism, where $S$ is a subspace of a metric space $(X, d)$. Let $W_{i}^{\rho}, 1 \leq i \leq n, \rho \in\{0,1\}$, be open sets in $(X, d)$ such that $W_{i}^{0} \cap W_{i}^{1}=\emptyset$ for all $i \in\{1, \ldots, n\}$. Let $\varepsilon>0$ be such that

$$
2 \varepsilon<d\left(f\left(A_{i}^{0}\right), f\left(A_{i}^{1}\right)\right)
$$

for each $i \in\{1, \ldots, n\}$. Suppose $C$ is an open spherical $\varepsilon-(n-1)$-chain in $(X, d)$ of length $m$ which is $\varepsilon$-proper, which covers $S$ and suppose that

$$
f\left(A_{i}^{\rho}\right) \subseteq W_{i}^{\rho}, \bigcup\left(\partial_{i}^{\rho} C\right) \subseteq W_{i}^{\rho}
$$

for all $i \in\{1, \ldots, n\}$ and $\rho \in\{0,1\}$. Then for each $x \in \bigcup C$ there exists $y \in S$ such that $d(x, y)<3 \varepsilon$.

Proof. It is enough to prove the following: for each $\left(p_{1}, \ldots, p_{n}\right) \in \partial \mathbb{N}_{m}^{n}$ with the property that $p_{k} \in\{0, m\}$ for exactly one $k \in\{1, \ldots, n\}$ the set $C_{p_{1}, \ldots, p_{n}}$ intersects $S$. Namely, if this holds, then for each $\left(q_{1}, \ldots, q_{n}\right) \in \partial \mathbb{N}_{m}^{n}$ there exists $\left(p_{1}, \ldots, p_{n}\right) \in \partial \mathbb{N}_{m}^{n}$ such that $\left|q_{1}-p_{1}\right| \leq 1, \ldots,\left|q_{n}-p_{n}\right| \leq 1$ and such that $C_{p_{1}, \ldots, p_{n}} \cap S \neq \emptyset$. Since $C$ is an $\varepsilon-(n-1)$ chain and $\varepsilon$-proper, we now easily get that for each $x \in \bigcup C$ there exists $y \in S$ such that $d(x, y)<3 \varepsilon$.

Suppose the opposite, that there exists $\left(p_{1}, \ldots, p_{n}\right) \in \partial \mathbb{N}_{m}^{n}$ such that $p_{k} \in\{0, m\}$ for exactly one $k \in\{1, \ldots, n\}$ and such that $C_{p_{1}, \ldots, p_{n}} \cap S=\emptyset$. We may assume $p_{n}=m$ (all other cases can be reduced to this one if we modify $C$ and $f$ by interchange of appropriate coordinates). It follows $0<p_{1}<m, \ldots, 0<p_{n-1}<m$.

For $i \in\{1, \ldots, n-1\}$ we define the set $U_{i}$ as the union of all sets of the following form:

$$
\begin{gathered}
C_{j_{1}, \ldots, j_{i-1}, l, j_{i+1}, \ldots, j_{n-1}, m}, \text { where } l<p_{i} ; \\
C_{j_{1}, \ldots, j_{i-1}, 0, j_{i+1}, \ldots, j_{n}} ;
\end{gathered}
$$

$$
C_{j_{1}, \ldots, j_{n-1}, 0} \text {, where this set is such that it intersects } f\left(A_{i}^{0}\right) .
$$

Furthermore, let $V_{i}$ be the union of all sets of the following form:

$$
\begin{gathered}
C_{j_{1}, \ldots, j_{i-1}, l, j_{i+1}, \ldots, j_{n-1}, m}, \text { where } l>p_{i} ; \\
C_{j_{1}, \ldots, j_{i-1}, m, j_{i+1}, \ldots, j_{n}} ; \\
C_{j_{1}, \ldots, j_{n-1}, 0} \text {, where this set is such that it intersects } f\left(A_{i}^{1}\right) .
\end{gathered}
$$

Let $i \in\{1, \ldots, n-1\}$. The sets $U_{i}$ and $V_{i}$ are open and it is straightforward to check that they are disjoint. (Figures 9 . and 10. show $C$ in case $n=3$ and $m=5$; the red set is $C_{p_{1}, p_{2}, p_{3}}$, in this case $C_{2,2,5}$, the blue sets in Figure 9 . are $U_{1}$ and $V_{1}$, the blue sets in Figure 10. are $U_{2}$ and $V_{2}$. For example, note that in Figure 11. the black set is $C_{3,0,2}$, the red set is $C_{5,0,1}$ and the blue set is $C_{5,0,5}$.) 


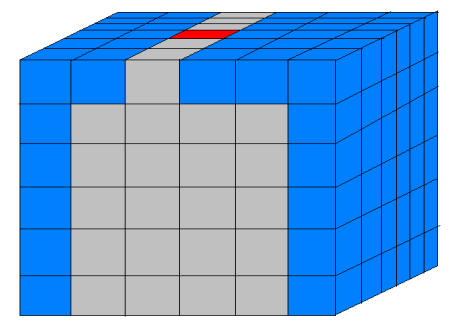

Figure 9.

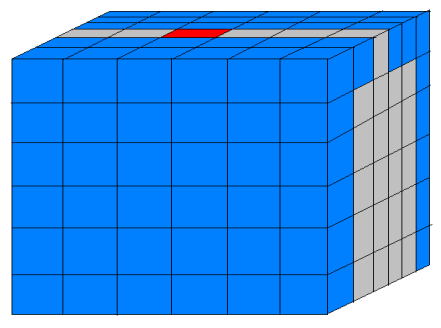

Figure 10.

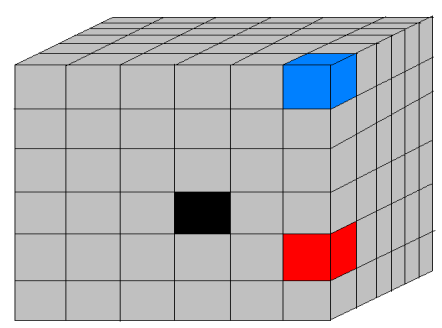

Figure 11.

We also have

$$
U_{i} \cap f\left(A_{i}^{1} \cap A_{n}^{0}\right)=\emptyset .
$$

Otherwise, the set $f\left(A_{i}^{1} \cap A_{n}^{0}\right)=f\left(A_{i}^{1}\right) \cap f\left(A_{n}^{0}\right)$ would intersect one of the sets in (3.7), (3.8) or (3.9). The sets in (3.7) are contained in $W_{n}^{1}$ which is disjoint with $f\left(A_{n}^{0}\right)$. The sets in (3.8) are contained in $W_{i}^{0}$ which is disjoint with $f\left(A_{i}^{1}\right)$. Finally, $f\left(A_{i}^{1}\right)$ cannot intersect a set in $(3.9)$ since $(3.6)$ holds. In the same way we get

$$
V_{i} \cap f\left(A_{i}^{0} \cap A_{n}^{0}\right)=\emptyset .
$$

Let

$$
\Omega=U_{1} \cup \cdots \cup U_{n-1} \cup V_{1} \cup \cdots \cup V_{n-1} .
$$

Let $i \in\{1, \ldots, n\}$ and $\rho \in\{0,1\}$ such that $(i, \rho) \neq(n, 0)$. We claim that

$$
f\left(A_{i}^{\rho}\right) \subseteq \Omega \text {. }
$$

Suppose that there exists $x \in f\left(A_{i}^{\rho}\right)$ such that $x \notin \Omega$. Since $C$ covers $S$, there exists $\left(j_{1}, \ldots, j_{n}\right) \in \partial \mathbb{N}_{m}^{n}$ such that

$$
x \in C_{j_{1}, \ldots, j_{n}} .
$$

We have $\left(j_{1}, \ldots, j_{n}\right) \neq\left(p_{1}, \ldots, p_{n}\right)$ since $C_{p_{1}, \ldots, p_{n}} \cap S=\emptyset$. So, if $j_{n}=m$, then $C_{j_{1}, \ldots, j_{n}}$ must be one of the sets in (3.7) or (3.10). But this is impossible since $x \notin \Omega$. So $j_{n}<m$. Now, if $j_{n}>0$, then $C_{j_{1}, \ldots, j_{n}}$ is one of the sets in (3.8) or (3.11), impossible. Therefore $j_{n}=0$.

We have $C_{j_{1}, \ldots, j_{n}} \cap f\left(A_{i}^{\rho}\right) \neq \emptyset$ and this also yields to contradiction. Namely, if $i<n$, then $C_{j_{1}, \ldots, j_{n}}$ is one of the sets in (3.9) or (3.12). And if $i=n$, then $\rho=1$ and

$$
f\left(A_{n}^{1}\right) \subseteq W_{n}^{1}, C_{j_{1}, \ldots, j_{n}} \subseteq \bigcup\left(\partial_{n}^{0} C\right) \subseteq W_{n}^{0},
$$

which is impossible since $W_{n}^{0} \cap W_{n}^{1}=\emptyset$. Hence 3.15 holds. 
Let

$$
E=A_{1}^{0} \cup \cdots \cup A_{n-1}^{0} \cup A_{1}^{1} \cup \cdots \cup A_{n}^{1} .
$$

For each $i \in\{1, \ldots, n-1\}$ the sets $f^{-1}\left(U_{i}\right)$ and $f^{-1}\left(V_{i}\right)$ are open in $\partial I^{n}$, they are disjoint, by $(3.13)$ and $(3.14)$

$$
f^{-1}\left(U_{i}\right) \cap\left(A_{i}^{1} \cap A_{n}^{0}\right)=\emptyset, f^{-1}\left(V_{i}\right) \cap\left(A_{i}^{0} \cap A_{n}^{0}\right)=\emptyset
$$

and by 3.15

$$
E \subseteq f^{-1}\left(U_{1}\right) \cup \cdots \cup f^{-1}\left(U_{n-1}\right) \cup f^{-1}\left(V_{1}\right) \cup \cdots \cup f^{-1}\left(V_{n-1}\right) .
$$

This is impossible by Corollary 3.3 .

The next proposition provides conditions under which an $n$-chain approximates an $n$ cell.

Proposition 3.9. Let $f: I^{n} \rightarrow E$ be a homeomorphism, where $E$ is a subspace of a metric space $(X, d)$. Suppose $C$ is an open $\varepsilon-n$-chain in $(X, d)$ of length $m$ which is $\varepsilon$-proper, which covers $E$ and such that $\partial C$ covers $f\left(\partial I^{n}\right)$ and suppose that $W_{i}^{\rho}, 1 \leq i \leq n, \rho \in\{0,1\}$, are open sets in $(X, d)$ such that

$$
\begin{gathered}
2 \varepsilon<d\left(W_{i}^{0}, W_{i}^{1}\right), \\
f\left(A_{i}^{\rho}\right) \subseteq W_{i}^{\rho} \text { and } \bigcup\left(\partial_{i}^{\rho} C\right) \subseteq W_{i}^{\rho}
\end{gathered}
$$

for all $i \in\{1, \ldots, n\}$ and $\rho \in\{0,1\}$. Then for each $x \in \bigcup C$ there exists $y \in E$ such that $d(x, y)<7 \varepsilon$.

Proof. It is enough to prove that for each $\left(p_{1}, \ldots, p_{n}\right) \in \mathbb{N}_{m}^{n}$ with the property that $1<$ $p_{k}<m-1$ for each $k \in\{1, \ldots, n\}$ the set $C_{p_{1}, \ldots, p_{n}}$ intersects $E$. Why is it enough to prove this? Suppose that this fact holds. Assume that $m \geq 4$. Let $x \in \bigcup C$. Then $x \in C_{q_{1}, \ldots, q_{n}}$ for some $\left(q_{1}, \ldots, q_{n}\right) \in \mathbb{N}_{m}^{n}$. For $i \in\{1, \ldots, n\}$ let

$$
q_{i}^{\prime}=\left\{\begin{array}{l}
1 \text { if } q_{i}=0, \\
m-1 \text { if } q_{i}=m, \\
q_{i} \text { otherwise }
\end{array}\right.
$$

Then $\left|q_{1}-q_{1}^{\prime}\right| \leq 1, \ldots,\left|q_{n}-q_{n}^{\prime}\right| \leq 1$ and $1 \leq q_{i}^{\prime} \leq m-1$ for each $i \in\{1, \ldots, n\}$. Since $C$ is $\varepsilon$-proper, there exist $x^{\prime} \in C_{q_{1}, \ldots, q_{n}}$ and $x^{\prime \prime} \in C_{q_{1}^{\prime}, \ldots, q_{n}^{\prime}}$ such that $d\left(x^{\prime}, x^{\prime \prime}\right)<\varepsilon$. Now, let the numbers $p_{1}, \ldots, p_{n}$ be defined by

$$
p_{i}=\left\{\begin{array}{l}
2 \text { if } q_{i}^{\prime}=1 \\
m-2 \text { if } q_{i}^{\prime}=m-1, \\
q_{i}^{\prime} \text { otherwise }
\end{array}\right.
$$

$i \in\{1, \ldots, n\}$. We have $\left|q_{1}^{\prime}-p_{1}\right| \leq 1, \ldots,\left|q_{n}^{\prime}-p_{n}\right| \leq 1 \mid$ and therefore there exist $y^{\prime \prime} \in C_{q_{1}^{\prime}, \ldots, q_{n}^{\prime}}$ and $y^{\prime} \in C_{p_{1}, \ldots, p_{n}}$ such that $d\left(y^{\prime \prime}, y^{\prime}\right)<\varepsilon$. Clearly $1<p_{i}<m-1$ for each $i \in\{1, \ldots, n\}$ and therefore there exists $y \in C_{p_{1}, \ldots, p_{n}} \cap E$. Using the fact that the diameters of the sets $C_{q_{1}, \ldots, q_{n}}, C_{q_{1}^{\prime}, \ldots, q_{n}^{\prime}}$ and $C_{p_{1}, \ldots, p_{n}}$ are less than $\varepsilon$, we obtain

$$
d(x, y) \leq d\left(x, x^{\prime}\right)+d\left(x^{\prime}, x^{\prime \prime}\right)+d\left(x^{\prime \prime}, y^{\prime \prime}\right)+d\left(y^{\prime \prime}, y^{\prime}\right)+d\left(y^{\prime}, y\right)<5 \varepsilon .
$$

If $m \leq 3$, then for all $\left(a_{1}, \ldots, a_{n}\right),\left(b_{1}, \ldots, b_{n}\right) \in \mathbb{N}_{m}^{n}$ we have, for each $i \in\{1, \ldots, n\}$, that $a_{i}, b_{i} \in\{0, \ldots, m\} \subseteq\{0,1,2,3\}$ and therefore there exist $c_{i}, d_{i} \in\{0, \ldots, m\}$ such that $\left|a_{i}-c_{i}\right| \leq 1,\left|c_{i}-d_{i}\right| \leq 1,\left|d_{i}-b_{i}\right| \leq 1$ which, together with the fact that $C$ is $\varepsilon$-proper, implies that there exist $x \in C_{a_{1}, \ldots, a_{n}}, x^{\prime}, y^{\prime} \in C_{c_{1}, \ldots, c_{n}}, y^{\prime \prime}, z^{\prime \prime} \in C_{d_{1}, \ldots, d_{n}}$ and $z \in C_{b_{1}, \ldots, b_{n}}$ 
such that $d\left(x, x^{\prime}\right)<\varepsilon, d\left(y^{\prime}, y^{\prime \prime}\right)<\varepsilon$ and $d\left(z^{\prime \prime}, z\right)<\varepsilon$. It follows $d(x, z)<5 \varepsilon$. This proves that $d\left(C_{a}, C_{b}\right)<5 \varepsilon$ for all $a, b \in \mathbb{N}_{m}^{n}$. Since $E$ is nonempty and contained in $\bigcup C$, there exists $b \in \mathbb{N}_{m}^{n}$ such that $C_{b} \cap E \neq \emptyset$. It follows $d\left(C_{a}, E\right)<6 \varepsilon$ for each $a \in \mathbb{N}_{m}^{n}$ and therefore for each $x \in \bigcup C$ we have $d(x, E)<7 \varepsilon$.

So, let $\left(p_{1}, \ldots, p_{n}\right) \in \mathbb{N}_{m}^{n}$ be such that $1<p_{k}<m-1$ for each $k \in\{1, \ldots, n\}$. We want to prove that $C_{p_{1}, \ldots, p_{n}} \cap E \neq \emptyset$. Suppose $C_{p_{1}, \ldots, p_{n}} \cap E=\emptyset$.

For $i \in\{1, \ldots, n\}$ let $U_{i}$ be the union of all sets $C_{j_{1}, \ldots, j_{n}}$ such that

$$
j_{i}<p_{i} \text { and } 1<j_{k}<m-1 \text { for all } k \in\{1, \ldots, n\}
$$

or

$$
j_{i} \in\{0,1\} .
$$

Let $V_{i}$ be the union of all sets $C_{j_{1}, \ldots, j_{n}}$ such that

$$
j_{i}>p_{i} \text { and } 1<j_{k}<m-1 \text { for all } k \in\{1, \ldots, n\}
$$

or

$$
j_{i} \in\{m-1, m\} .
$$

For each $i \in\{1, \ldots, n\}$ the sets $U_{i}$ and $V_{i}$ are open and disjoint. Note that every $C_{j_{1}, \ldots, j_{n}}$, where $\left(j_{1}, \ldots, j_{n}\right) \neq\left(p_{1}, \ldots, p_{n}\right)$, is contained in some $U_{i}$ or $V_{i}$. Therefore

$$
E \subseteq U_{1} \cup \cdots \cup U_{n} \cup V_{1} \cup \cdots \cup V_{n} .
$$

Let $i \in\{1, \ldots, n\}$. We prove now that

$$
U_{i} \cap f\left(A_{i}^{1}\right)=\emptyset .
$$

Suppose the opposite, that $U_{i} \cap f\left(A_{i}^{1}\right) \neq \emptyset$. It follows from the definition of $U_{i}$ that there exist $j_{1}, \ldots, j_{n} \in \mathbb{N}_{m}$ such that 3.16 or 3.17 hold and such that $C_{j_{1}, \ldots, j_{n}} \cap f\left(A_{i}^{1}\right) \neq \emptyset$. However, if (3.16) holds, then $1<j_{k}<m-1$ for all $k \in\{1, \ldots, n\}$ which implies that $C_{j_{1}, \ldots, j_{n}}$ is disjoint with $\bigcup(\partial C)$. But we have the assumption that $\partial C$ covers $f\left(\partial I^{n}\right)$ and this implies that $C_{j_{1}, \ldots, j_{n}}$ is disjoint with $f\left(\partial I^{n}\right)$ which is impossible. Therefore, (3.16) does not hold which means that (3.17) holds. Hence we have

$$
C_{j_{1}, \ldots, j_{n}} \cap f\left(A_{i}^{1}\right) \neq \emptyset \text { and } j_{i} \in\{0,1\} .
$$

This, together with $\bigcup\left(\partial_{i}^{0} C\right) \subseteq W_{i}^{0}$ and $f\left(A_{i}^{1}\right) \subseteq W_{i}^{1}$, implies $d\left(W_{i}^{0}, W_{i}^{1}\right)<2 \varepsilon$ (namely, if $j_{i}=0$, then $W_{i}^{0} \cap W_{i}^{1} \neq \emptyset$, and if $j_{i}=1$, then $d\left(C_{j_{1}, \ldots, j_{n}}, W_{i}^{0}\right)<\varepsilon$ since $C$ is $\varepsilon$-proper and this implies $\left.d\left(W_{i}^{0}, W_{i}^{1}\right)<2 \varepsilon\right)$. A contradiction. Hence 3.19 holds. In the same way we get

$$
V_{i} \cap f\left(A_{i}^{0}\right)=\emptyset .
$$
3.18

For each $i \in\{1, \ldots, n\}$ the sets $f^{-1}\left(U_{i}\right)$ and $f^{-1}\left(V_{i}\right)$ are open in $I^{n}$ and disjoint. By

$$
I^{n}=f^{-1}\left(U_{1}\right) \cup \cdots \cup f^{-1}\left(U_{n}\right) \cup f^{-1}\left(V_{1}\right) \cup \cdots \cup f^{-1}\left(V_{n}\right),
$$

and by 3.19 and 3.20

$$
f^{-1}\left(U_{i}\right) \cap A_{i}^{1}=\emptyset, f^{-1}\left(V_{i}\right) \cap A_{i}^{0}=\emptyset .
$$

This is impossible by Corollary 3.2 . 


\section{Computability of CO-C.E. Spheres AND Cells}

Let $n \geq 1$. A finite $n$-sequence in $\mathbb{N}$ is any function of the form

$$
\{0, \ldots, m\}^{n} \rightarrow \mathbb{N}
$$

Recall that any finite sequence $i_{0}, \ldots, i_{m}$ in $\mathbb{N}$ is of the form $(j)_{0}, \ldots,(j)_{\bar{j}}$ for some $j \in \mathbb{N}$. Let $f: \mathbb{N}^{n} \rightarrow \mathbb{N}$ be some computable injection and let $\tau$ and $\tau^{\prime}$ be the functions from the section Preliminaries. We define $\Sigma: \mathbb{N}^{n+1} \rightarrow \mathbb{N}$ by

$$
\Sigma\left(i, j_{1}, \ldots, j_{n}\right)=(\tau(i))_{f\left(j_{1}, \ldots, j_{n}\right)} .
$$

Then for any finite $n$-sequence $a$ in $\mathbb{N}$ there exists $i \in \mathbb{N}$ such that $a$ equals the function

$$
\begin{gathered}
\left\{0, \ldots, \tau^{\prime}(i)\right\}^{n} \rightarrow \mathbb{N}, \\
\left(j_{1}, \ldots, j_{n}\right) \mapsto \Sigma\left(i, j_{1}, \ldots, j_{n}\right) .
\end{gathered}
$$

We will use the following notation: $\widehat{i}$ instead of $\tau^{\prime}(i)$ and, for $n \geq 2,(i)_{j_{1}, \ldots, j_{n}}$ instead of $\Sigma\left(i, j_{1}, \ldots, j_{n}\right)$.

Let $(X, d, \alpha)$ be a computable metric space. For $l \in \mathbb{N}$ let $\mathcal{H}_{l}$ be the finite $n$-sequence of sets in $X$ defined by

$$
\mathcal{H}_{l}=\left(J_{(l)_{j_{1}, \ldots, j_{n}}}\right)_{0 \leq j_{1}, \ldots, j_{n} \leq \widehat{l}}
$$

(i.e. $\mathcal{H}_{l}$ is the function $\left\{0, \ldots, \widehat{l}^{n} \rightarrow \mathcal{P}(X)\right.$ which maps $\left(j_{1}, \ldots, j_{n}\right)$ to $\left.J_{(l)_{j_{1}, \ldots, j_{n}}}\right)$.

For $l \in \mathbb{N}$ let $\widehat{\mathcal{H}}_{l}$ be defined by

$$
\widehat{\mathcal{H}}_{l}=\left(\widehat{J}_{(l)_{j_{1}, \ldots, j_{n}}}\right)_{0 \leq j_{1}, \ldots, j_{n} \leq \widehat{l}} .
$$

In Euclidean space $\mathbb{R}^{n}$ we can effectively calculate the diameter of the finite union of rational balls. However, in a general computable metric space the function $\mathbb{N} \rightarrow \mathbb{R}$, $j \mapsto \operatorname{diam}\left(J_{j}\right)$, need not be computable. For that reason we are going to use the notion of the formal diameter. Let $(X, d)$ be a metric space and $x_{0}, \ldots, x_{k} \in X, r_{0}, \ldots, r_{k} \in \mathbb{R}_{+}$. The formal diameter associated to the finite sequence $\left(x_{0}, r_{0}\right), \ldots,\left(x_{k}, r_{k}\right)$ is the number $D \in \mathbb{R}$ defined by

$$
D=\max _{0 \leq v, w \leq k} d\left(x_{v}, x_{w}\right)+2 \max _{0 \leq v \leq k} r_{v} .
$$

Let $(X, d, \alpha)$ be a computable metric space. We define the function fdiam $: \mathbb{N} \rightarrow \mathbb{R}$ in the following way. For $j \in \mathbb{N}$ the number $\operatorname{fdiam}(j)$ is the formal diameter associated to the finite sequence

$$
\left(\alpha_{\tau\left((j)_{0}\right)}, q_{\tau^{\prime}\left((j)_{0}\right)}\right), \ldots,\left(\alpha_{\tau\left((j)_{\bar{j}}\right)}, q_{\tau^{\prime}\left((j)_{\bar{j}}\right)}\right) .
$$

We have the following proposition (for the proof see [5]).

Proposition 4.1. Let $(X, d, \alpha)$ be a computable metric space.

(1) For all $j \in \mathbb{N}$, $\operatorname{diam}\left(\widehat{J}_{j}\right) \leq \operatorname{fdiam}(j)$.

(2) fdiam $: \mathbb{N} \rightarrow \mathbb{R}$ is a computable function.

(3) Let $S$ be a compact subset of $(X, d), r \in \mathbb{R}_{+}$and $C_{0}, \ldots, C_{m}$ a finite sequence of open sets which covers $S$ and such that $\operatorname{diam}\left(C_{i}\right)<r$ for each $i \in\{0, \ldots, m\}$. Then there exist $j_{0}, \ldots, j_{m} \in \mathbb{N}$ such that the finite sequence of sets $J_{j_{0}}, \ldots, J_{j_{m}}$ covers $S, \widehat{J_{j_{i}}} \subseteq C_{i}$ and $\operatorname{fdiam}\left(j_{i}\right)<r$ for each $i \in\{0, \ldots, m\}$. 
Let the function fmesh $: \mathbb{N} \rightarrow \mathbb{R}$ be defined by

$$
\operatorname{fmesh}(l)=\max _{0 \leq j_{1}, \ldots, j_{n} \leq \widehat{l}} \operatorname{fdiam}\left((l)_{j_{1}, \ldots, j_{n}}\right) .
$$

It is immediate from Proposition 4.1 and Proposition 2.2 that fmesh is a computable function.

Proposition 4.2. Let $(X, d, \alpha)$ be a computable metric space. The sets

$$
\Omega=\left\{(l, k) \in \mathbb{N}^{2} \mid \mathcal{H}_{l} \text { is } 2^{-k} \text { - proper }\right\}
$$

and

$$
\Omega^{\prime}=\left\{(l, k) \in \mathbb{N}^{2} \mid \partial \mathcal{H}_{l} \text { is } 2^{-k}-\text { proper }\right\}
$$

are c.e.

Proof. Let $\Phi: \mathbb{N}^{2} \rightarrow \mathcal{P}\left(\mathbb{N}^{2 n+2}\right)$ be defined in the following way. For $l, k \in \mathbb{N}$ let $\Phi(l, k)$ be the set of all

$$
\left(l, k, i_{1}, . ., i_{n}, j_{1}, \ldots, j_{n}\right)
$$

such that $i_{1}, . ., i_{n}, j_{1}, \ldots, j_{n} \in \mathbb{N}_{\widehat{l}}$ and $\left|i_{1}-j_{1}\right| \leq 1, \ldots,\left|i_{n}-j_{n}\right| \leq 1$. Then $\Phi$ is c.c.b. On the other hand, let $S$ be the set of all $\left(l, k, i_{1}, \ldots, i_{n}, j_{1}, \ldots, j_{n}\right)$ for which there exists $x \in J_{(l)_{i_{1}, \ldots, i_{n}}}$ and $y \in J_{(l)_{j_{1}, \ldots, j_{n}}}$ such that $d(x, y)<2^{-k}$. This is equivalent to the fact that there exist $p, q \in \mathbb{N}$ such that

$$
\alpha_{p} \in J_{(l)_{i_{1}, \ldots, i_{n}}}, \alpha_{q} \in J_{(l)_{j_{1}, \ldots, j_{n}}} \text { and } d\left(\alpha_{p}, \alpha_{q}\right)<2^{-k}
$$

The set $T$ of all $\left(l, k, i_{1}, \ldots, i_{n}, j_{1}, \ldots, j_{n}, p, q\right)$ such that (4.1) holds is c.e. by Corollary 2.4 and Proposition 2.2. Therefore $S$ is c.e. Since

$$
\Omega=\{(l, k) \mid \Phi(l, k) \subseteq S\}
$$

we have that $\Omega$ is c.e. by Proposition 2.1. We similarly get that $\Omega^{\prime}$ is c.e.

Lemma 4.3. Let $(X, d, \alpha)$ be a computable metric space. There exists a computable function $\zeta: \mathbb{N} \rightarrow \mathbb{N}$ such that $J_{\zeta(l)}=\bigcup \mathcal{H}_{l}$ for each $l \in \mathbb{N}$. There exists a computable function $\zeta^{\prime}: \mathbb{N} \rightarrow \mathbb{N}$ such that $J_{\zeta^{\prime}(l)}=\bigcup\left(\partial \mathcal{H}_{l}\right)$ for each $l \in \mathbb{N}$. Furthermore, for all $i \in\{1, \ldots, m\}$ and $\rho \in\{0,1\}$ there exists a computable function $\zeta^{\prime \prime}: \mathbb{N} \rightarrow \mathbb{N}$ such that $J_{\zeta^{\prime \prime}(l)}=\bigcup\left(\partial_{i}^{\rho} \mathcal{H}_{l}\right)$ for each $l \in \mathbb{N}$. Similar statements hold for $\widehat{J}_{j}$ and $\widehat{\mathcal{H}}_{l}, \partial \widehat{\mathcal{H}}_{l}, \partial_{i}^{\rho} \widehat{\mathcal{H}}_{l}$.

Proof. It is enough to prove the following: if $\Phi: \mathbb{N} \rightarrow \mathcal{P}\left(\mathbb{N}^{n}\right)$ and $\Psi: \mathbb{N}^{n} \rightarrow \mathcal{P}(\mathbb{N})$ are c.c.b. functions such that $\Phi(l) \neq \emptyset$ and $\Psi(a) \neq \emptyset$ for all $l \in \mathbb{N}$ and $a \in \mathbb{N}^{n}$, then there exists a computable function $\zeta: \mathbb{N} \rightarrow \mathbb{N}$ such that

$$
J_{\zeta(l)}=\bigcup_{a \in \Phi(l)} \bigcup_{i \in \Psi(a)} I_{i}
$$

However, if $\Phi$ and $\Psi$ are such functions, by Proposition 2.1 there exists a c.c.b. function $\Lambda: \mathbb{N} \rightarrow \mathcal{P}(\mathbb{N})$ such that

$$
\bigcup_{i \in \Lambda(l)} I_{i}=\bigcup_{a \in \Phi(l)} \bigcup_{i \in \Psi(a)} I_{i}
$$

For each $l \in \mathbb{N}$ there exists $j \in \mathbb{N}$ such that $\Lambda(l)=[j]$ (recall definition (2.1)). Since the set $S=\{(l, j) \mid \Lambda(l)=[j]\}$ is computable (Proposition 2.1) and for each $l \in \mathbb{N}$ there exists $j \in \mathbb{N}$ such that $(l, j) \in S$, there exists a computable function $\zeta: \mathbb{N} \rightarrow \mathbb{N}$ such that $(l, \zeta(l)) \in S$ for each $l \in \mathbb{N}$. It follows 4.2 . 
The proof of the following proposition can be found in [5].

Proposition 4.4. Let $(X, d, \alpha)$ be a computable metric space which has the effective covering property and compact closed balls.

(1) The set $\left\{(i, j) \in \mathbb{N}^{2} \mid \widehat{J}_{i} \subseteq J_{j}\right\}$ is c.e.

(2) Let $S$ be a co-c.e. set in $(X, d, \alpha)$ which is compact. Then the set $\left\{j \in \mathbb{N} \mid S \subseteq J_{j}\right\}$ is c.e.

Corollary 4.5. Let $(X, d, \alpha)$ be a computable metric space which has the effective covering property and compact closed balls and let $S$ be a co-c.e. set in $(X, d, \alpha)$ which is compact. Then the sets

$$
\left\{l \in \mathbb{N} \mid \mathcal{H}_{l} \text { covers } S\right\} \text { and }\left\{l \in \mathbb{N} \mid \partial \mathcal{H}_{l} \text { covers } S\right\}
$$

are c.e.

Proof. This follows from Lemma 4.3 and Proposition 4.4 .

The following proposition can be proved in the same way as Proposition 32 in [5].

Proposition 4.6. Let $(X, d, \alpha)$ be a computable metric space which has the effective covering property and compact closed balls. The sets $\Omega=\left\{l \in \mathbb{N} \mid \widehat{\mathcal{H}}_{l}\right.$ is an $n$-chain $\}$ and $\Omega^{\prime}=\left\{l \in \mathbb{N} \mid \partial \widehat{\mathcal{H}}_{l}\right.$ is a spherical $(n-1)$-chain $\}$ are computably enumerable.

The following lemma can be proved similarly as Lemma 14 in [5].

Lemma 4.7. Let $(X, d, \alpha)$ be a computable metric space. Let $S$ be a compact set in this space such that that there exists a computable function $f: \mathbb{N} \rightarrow \mathbb{N}$ with the property that for each $k \in \mathbb{N}$ the following holds:

$$
S \subseteq J_{f(k)} \text { and for each } x \in J_{f(k)} \text { there exists } y \in S \text { such that } d(x, y)<2^{-k} .
$$

Then $S$ is computable.

Theorem 4.8. Let $(X, d, \alpha)$ be a computable metric space which is locally computable. Let $S$ be an $(n-1)$-sphere in $(X, d)$ and suppose $S$ is co-c.e. in $(X, d, \alpha)$. Then $S$ is computable.

Proof. As we have seen, we may assume that $(X, d, \alpha)$ has compact closed balls and the effective covering property. Let $f: \partial I^{n} \rightarrow S$ be a homeomorphism. Choose sets $W_{i}^{\rho}$, $1 \leq i \leq n, \rho \in\{0,1\}$, so that each of these sets is a finite union of rational balls (i.e. of the form $J_{j}$ ) and so that

$$
W_{i}^{0} \cap W_{i}^{1}=\emptyset \text { and } f\left(A_{i}^{\rho}\right) \subseteq W_{i}^{\rho}
$$

for all $i \in\{1, \ldots, n\}$ and $\rho \in\{0,1\}$.

Let $k_{0} \in \mathbb{N}$ be such that

$$
2 \cdot 2^{-k_{0}}<d\left(f\left(A_{i}^{0}\right), f\left(A_{i}^{1}\right)\right)
$$

for each $i \in\{1, \ldots, n\}$.

By Proposition 3.6 for each $\varepsilon>0$ there exists an open spherical $\varepsilon-(n-1)$-chain $C$ in $(X, d)$ which is $\varepsilon$-proper, which covers $S$ and such that

$$
f\left(A_{i}^{\rho}\right) \subseteq \bigcup\left(\partial_{i}^{\rho} C\right) \subseteq W_{i}^{\rho}
$$

for all $i \in\{1, \ldots, n\}$ and $\rho \in\{0,1\}$. 
From this, Lemma 3.4 and Proposition 4.1 we conclude that for each $k \in \mathbb{N}$ there exists $l \in \mathbb{N}$ with the following properties:

$$
\begin{gathered}
\partial \widehat{\mathcal{H}}_{l} \text { is a spherical }(n-1) \text {-chain, } \\
\partial \mathcal{H}_{l} \text { covers } S \\
\operatorname{fmesh}(l)<2^{-\left(k+k_{0}\right)} \\
\partial \mathcal{H}_{l} \text { is } 2^{-\left(k+k_{0}\right)}-\text { proper }
\end{gathered}
$$

and

$$
\bigcup\left(\partial_{i}^{\rho} \widehat{\mathcal{H}}_{l}\right) \subseteq W_{i}^{\rho}
$$

for all $i \in\{1, \ldots, n\}$ and $\rho \in\{0,1\}$.

Let $\Omega$ be the set of all $(k, l)$ such that (4.3), (4.4), (4.5), (4.6) and (4.7) hold. Then $\Omega$ is c.e., which follows from Proposition 4.6, Corollary 4.5, Proposition 4.2, Lemma 4.3 , Proposition 4.4(1) and the fact that fmesh is a computable function. The fact that $\Omega$ is c.e. and the fact that for each $k \in \mathbb{N}$ there exists $l \in \mathbb{N}$ such that $(k, l) \in \Omega$ imply that there exists a computable function $g: \mathbb{N} \rightarrow \mathbb{N}$ such that $(k, g(k)) \in \Omega$ for each $k \in \mathbb{N}$.

Let $k \in \mathbb{N}$. By Proposition 3.8 for each $x \in \bigcup\left(\partial \mathcal{H}_{g(k)}\right)$ there exists $y \in S$ such that $d(x, y)<3 \cdot 2^{-k}$. Now Lemma 4.3 and Lemma 4.7 imply that $S$ is computable.

Theorem 4.9. Let $(X, d, \alpha)$ be a computable metric space which is locally computable. Let $E$ be an $n$-cell in $(X, d)$ and suppose $E$ and the boundary sphere of $E$ are co-c.e. in $(X, d, \alpha)$. Then $E$ is computable.

Proof. We proceed in a similar way as in the proof of Theorem 4.8. First, we may assume that $(X, d, \alpha)$ has compact closed balls and the effective covering property. Let $f: I^{n} \rightarrow E$ be a homeomorphism. Let $S=f\left(\partial I^{n}\right)$. Choose sets $W_{i}^{\rho}, 1 \leq i \leq n, \rho \in\{0,1\}$, so that each of these sets is a finite union of rational balls and so that the closures $\overline{W_{i}^{0}}$ and $\overline{W_{i}^{1}}$ are disjoint and $f\left(A_{i}^{\rho}\right) \subseteq W_{i}^{\rho}$ for all $i \in\{1, \ldots, n\}$ and $\rho \in\{0,1\}$. Let $k_{0} \in \mathbb{N}$ be such that $2 \cdot 2^{-k_{0}}<d\left(W_{i}^{0}, W_{i}^{1}\right)$ for each $i \in\{1, \ldots, n\}$ (such $k_{0}$ certainly exists since $\overline{W_{i}^{0}}$ and $\overline{W_{i}^{1}}$ are compact and disjoint for each $i \in\{1, \ldots, n\})$.

Using Proposition 3.7, Lemma 3.4 and Proposition 4.1 we conclude that for each $k \in \mathbb{N}$ there exists $l \in \mathbb{N}$ with the following properties:

$$
\begin{aligned}
& \widehat{\mathcal{H}}_{l} \text { is an } n \text {-chain, } \mathcal{H}_{l} \text { covers } E, \partial \mathcal{H}_{l} \text { covers } S, \\
& \text { fmesh }(l)<2^{-\left(k+k_{0}\right)}, \mathcal{H}_{l} \text { is } 2^{-\left(k+k_{0}\right)}-\text { proper }
\end{aligned}
$$

and

$$
\bigcup\left(\partial_{i}^{\rho} \widehat{\mathcal{H}}_{l}\right) \subseteq W_{i}^{\rho}
$$

for all $i \in\{1, \ldots, n\}$ and $\rho \in\{0,1\}$.

As in the proof of Theorem 4.8 we conclude that there exists a computable function $g: \mathbb{N} \rightarrow \mathbb{N}$ such that (4.8), 4.9) and 4.10) hold for each $k \in \mathbb{N}$ and $l=g(k)$. Let $k \in \mathbb{N}$. By Proposition 3.9 for each $x \in \cup \mathcal{H}_{g(k)}$ there exists $y \in E$ such that $d(x, y)<7 \cdot 2^{-k}$ and therefore $E$ is computable. 
Let us mention that a computable $n$-cell need not be computably homeomorphic to the unit ball in $\mathbb{R}^{n}$. It has been shown in 7 that there exists a computable arc $E$ in $\mathbb{R}^{2}$ with computable endpoints, but such that there exists no homomorphism $[0,1] \rightarrow E$ which is a computable function. Similarly, a computable $(n-1)$-sphere need not be computably homeomorphic to the unit sphere in $\mathbb{R}^{n}([7])$.

\section{Conclusion}

In this paper we have seen that topology plays an important role regarding the computability of co-c.e. sets in computable metric space. We have seen that the topological types of an arbitrary dimensional sphere and an arbitrary dimensional cell behave well from this viewpoint not just in Euclidean space but in any computable metric space which is locally computable, in particular in any computable metric space which has the effective covering property and which is locally compact. Such a computable metric space is for example the Hilbert cube $I^{\infty}$, equipped with a natural computability structure (see e.g. [5]).

It should be mentioned that co-c.e. spheres, as well as co-c.e. cells with co-c.e. boundary spheres, need not be computable in a computable metric space which is not locally computable. Moreover, by [6], there are examples of computable metric spaces $X$ and $Y$ such that $X$ has the effective covering property and $Y$ is compact, but such that both $X$ and $Y$ have noncomputable co-c.e. topological circles and a noncomputable co-c.e. arcs with computable endpoints.

\section{ACKNOWLEDGEMENTS}

The author would like to thank the anonymous referees for their careful work and many useful suggestions and corrections. Furthermore, the author is grateful to Professor Sibe Mardešić and Professor Ivan Ivanšić for their helpful comments.

\section{REFERENCES}

[1] Vasco Brattka. Plottable real number functions and the computable graph theorem. SIAM J. Comput., 38(1):303-328, 2008.

[2] Vasco Brattka and Gero Presser. Computability on subsets of metric spaces. Theoretical Computer Science, 305:43-76, 2003.

[3] Charles O. Christenson and William L. Voxman. Aspects of Topology. Marcel Dekker, Inc., New York, 1977.

[4] Ryszard Engelking. Dimension Theory. PWN - Polish Scientific Publishers, Warszawa, 1978.

[5] Zvonko Iljazović. Chainable and Circularly Chainable Co-c.e. Sets in Computable Metric Spaces. Journal of Universal Computer Science, 15(6):1206-1235, 2009.

[6] Zvonko Iljazović. Local Computability of Computable Metric Spaces and Computability of Co-c.e. Continua. To appear in Glasnik matematički.

[7] Joseph S. Miller. Effectiveness for Embedded Spheres and Balls. Electronic Notes in Theoretical Computer Science, 66:127-138, 2002.

[8] James R. Munkres. Elements of Algebraic Topology. Addison-Wesley Publishing Company, Menlo Park, California, 1984.

[9] Ernst Specker. Der Satz vom Maximum in der rekursiven Analysis. Constructivity in Mathematics (A. Heyting, ed.). North Holland Publ. Comp., Amsterdam, 254-265, 1959.

[10] Klaus Weihrauch. Computable Analysis Springer, Berlin, 2000.

This work is licensed under the Creative Commons Attribution-NoDerivs License. To view a copy of this license, visit http://creativecommons.org/licenses/by-nd/2.0/ or send a letter to Creative Commons, 171 Second St, Suite 300, San Francisco, CA 94105, USA, or Eisenacher Strasse 2, 10777 Berlin, Germany 